\title{
Harvesting Energy Using Triboelectric Nanogenerator Mounted inside Rolling Tire
}

\author{
Hiroshi Tani, ${ }^{1 *}$ Mutsuki Sugimoto, ${ }^{2}$ Kazuhisa Fushihara, ${ }^{2}$ Yukio Nakano, ${ }^{2}$ \\ Renguo Lu, ${ }^{1}$ Shinji Koganezawa, ${ }^{1}$ and Norio Tagawa ${ }^{1}$ \\ ${ }^{1}$ Mechanical Engineering Department, Kansai University, 3-3-35 Yamate-cho, Suita-shi, Osaka 564-8680, Japan \\ ${ }^{2}$ Sumitomo Rubber Industries, Ltd., 2-1-1 Tsutsui-cho, Chuo-ku, Kobe, Hyogo 651-0072, Japan
}

(Received February 10, 2020; accepted March 9, 2020)

Keywords: triboelectric nanogenerator, triboelectrification, tire, energy harvesting

In this study, we developed a triboelectric nanogenerator (TENG) for intelligent tires. This TENG was attached on the inner surface of a racing cart tire. First, we evaluated the dependences of the TENG output on the environmental temperature and relative humidity to ensure that the proposed TENG can operate in a wide range of these parameters. Then, we packaged the TENGs in aluminum laminate film and mounted them on the inner side of the tire. The charged voltage and output voltage of the TENGs were evaluated on a moving belt. The output voltage signals were observed at the region of contact; the signals increased with rotational speed and load weight but decreased with tire pressure. The charged voltage in the $10 \mu \mathrm{F}$ capacitor was found to be approximately $14 \mathrm{~V}$, which corresponds to $1.0 \mathrm{~mJ}$ at the speed of $16 \mathrm{~km} / \mathrm{h}$ after the tire rotated for $15 \mathrm{~min}$. These results suggest that the proposed TENG can operate as an energy harvester for the temperature and pressure sensors built into intelligent tires.

\section{Introduction}

The tread of a vehicle tire is the only component that interacts with the road surface. It dictates the motion of the vehicle and is an important factor in the stability and control of the vehicle. Intelligent tires with tire condition monitoring systems (TCMSs) that can monitor parameters such as the coefficient of friction between tires and the road surface, vehicle speed, tire pressure, and tire load are being developed by various companies. Tire pressure monitoring systems (TPMSs) have already been installed to observe tire pressure. In the future, TCMSs mounted on tires will play an important role in adaptively adjusting the vehicle stability. Power components are a significant part of the TCMS. Therefore, TCMSs require an energy harvester that can operate in severe tire environments, which are already significantly harsh because their range of operating temperature and humidity is -40 to $130{ }^{\circ} \mathrm{C}^{(1)}$ and $0-100 \%$ relative humidity $(\mathrm{RH})$, respectively. Moreover, the acceleration in an energy harvester is very large. Additionally, the energy harvester should be lightweight to ensure rotational balance in the tires.

*Corresponding author: e-mail: hrstani@kansai-u.ac.jp

https://doi.org/10.18494/SAM.2020.2827 
Several studies have been conducted to develop energy harvesters for TCMSs. Several researchers have focused on the development of piezoelectric energy harvesters for TCMSs. Keck developed an energy harvester using piezoelectric materials to provide power to the sensors embedded in a tire. ${ }^{(2)}$ Their energy-harvesting device could generate an average power of $40 \mu \mathrm{W}$ over a velocity range of $30-180 \mathrm{~km} / \mathrm{h}$. Zheng et al. proposed a piezoelectric energy-harvesting device that should be installed on the wheel upside down. This device works in the compression mode. ${ }^{(3)}$ Their energy-harvesting device could scavenge $47 \mu \mathrm{W}$ at approximately $80 \mathrm{~km} / \mathrm{h}$. Tang et al. developed an energy-harvesting device with piezoelectric cantilevers excited by magnetic repulsive force. ${ }^{(4)}$ This device was mounted on the rim inside the tire cavity and effectively generated an average power of $10 \mu \mathrm{W}$. Lee and Choi developed a piezoelectric energy harvester that generated $380.2 \mu \mathrm{J}$ per revolution under a load of $500 \mathrm{kgf}$ at a velocity of $60 \mathrm{~km} / \mathrm{h}^{(5)}$ They fabricated a self-powered wireless sensor system for application and tested it during driving of a vehicle. The harvester generated a power of $1.37 \mu \mathrm{W} / \mathrm{mm}^{3}$. Singh et al. proposed a novel artificial neural-network-based closed-loop system capable of ensuring broadband operations to optimize the harvester performance with changing dominant tire vibration frequencies. ${ }^{(6)}$

Electromagnetic energy harvesters have been considered viable devices for the TCMSs. ${ }^{(7)}$ Inertial electromagnetic and relative displacement electromagnetic transducers are the two different types of electromagnetic energy-harvesting devices in tires. Wang et al. proposed an energy harvester to harvest power from a rotating wheel. ${ }^{(8)}$ Their fabricated harvester includes two springs along with a coil, a magnet, and an electrical circuit. Their device could generate a power of 30-4200 $\mu \mathrm{W}$ for a range of speed between 200 and $900 \mathrm{rpm}$. Wang et al. also developed an energy harvester using a well-weighted pendulum to harvest energy from a rotating wheel by the electromagnetic induction technique. ${ }^{(9)}$ Their device could generate several milliwatts, which is sufficient for operating sensors inside a tire. Wang et al. then proposed a similar design using a weighted pendulum-type electromagnetic harvester attached to a rotating wheel. ${ }^{(10)}$ Their energy harvester could generate an electrical power of $200-300 \mu \mathrm{W}$ at approximately $200-400 \mathrm{rpm}$.

Mao et al. utilized single-electrode triboelectric nanogenerators (TENGs) using triboelectrification to scavenge the friction energy from rolling tires. ${ }^{(11)}$ They systematically analyzed the potential of TENGs in tires. Their fabricated device could generate a maximum instantaneous power xof $1.79 \mathrm{~mW}$ at a load resistance of $10 \mathrm{M} \Omega$. Qian et al. proposed a magnetic TENG mounted on the wheel hub of a tire for self-powered TPMS application. Their TENG could deliver a maximal output voltage of $316 \mathrm{~V}$ and power of $22.3 \mathrm{~mW}$ at a rotation speed of $100 \mathrm{rpm}$ and load of $3 \mathrm{M} \Omega$, thereby enabling the self-powered sensor to monitor the tire pressure. ${ }^{(12)}$ Guo et al. demonstrated an array of compressible hexagonal TENGs for harvesting the mechanical energy from a rolling tire. A combination of 8 units of TENGs yielded a maximum instantaneous power of $1.9 \mathrm{~mW}$ at a weight load of $10 \mathrm{~N}$ and speed of $2.51 \mathrm{~m} / \mathrm{s}$; additionally, it was found to be stable and durable after 30 continuous working days and could power a wireless tire pressure sensor once every $14 \mathrm{~min}$ at a rolling speed of $2.51 \mathrm{~m} / \mathrm{s}$ and load weight of $10 \mathrm{~N} .^{(13)}$ Wu et al. developed a smart green-energy tire by introducing silica fillers and TENGs into natural rubber tires. This green-energy tire could serve as a self- 
powered sensor to monitor subtle changes in the pressure and road condition from the TENG output signal. $^{(14)}$

In contrast, the TENG output is affected by the environmental humidity because the charged static electricity in the contact surfaces of the TENG escapes into the air owing to the environmental humidity. Shen et al. studied humidity-resistant TENGs for high-performance biomechanical energy harvesting. ${ }^{(15)}$ They applied electrospun nanofibrous membranes in the TENG to eliminate the adverse effects of water vapor on the electrical output. Mule et al. proposed a pouch-type TENG to significantly reduce the effect of $\mathrm{RH} .{ }^{(16)}$

Generally, the TENG is mounted in the closed space of tires. It is used in a wide range of temperatures. Additionally, the humidity in tires varies from 0 to $100 \% \mathrm{RH}$. Thus, we developed a TENG using a silicone-rubber base sheet with cylindrical protrusions that contributed to the roughness of the surface. ${ }^{(17)}$ Our TENG was mounted inside the rolling tire and the TENG output signal was confirmed at different rolling speeds, tire pressures, and load weights. ${ }^{(18)}$ We assert that our TENG is a suitable energy harvester for mounting on a rolling tire, because it was indeed easily mounted and the power was harvested from the deformation of TENG at the contact patch of the rolling tire, which was the portion of a vehicle's tire that was in actual contact with the road surface. In addition, the cost and weight of our TENG are considerably low. In this study, we designed a packaged TENG, mounted it in tires, and confirmed its output at different rolling speeds, tire pressures, and load weights.

\section{Experimental Sample and Setup}

\subsection{Structure of TENG}

The structure of the contact-type TENG is illustrated in Fig. 1. When two different materials come into contact, the transfer of electrons between the material surfaces causes contact electrification. In this study, we used fluorinated ethylene propylene (FEP) and polyurethane (PU) films. After the silicone rubber surface underwent plasma etching, a conductive cloth electrode was adhered to the silicone rubber. An FEP film with a thickness of $12.5 \mu \mathrm{m}$ and a

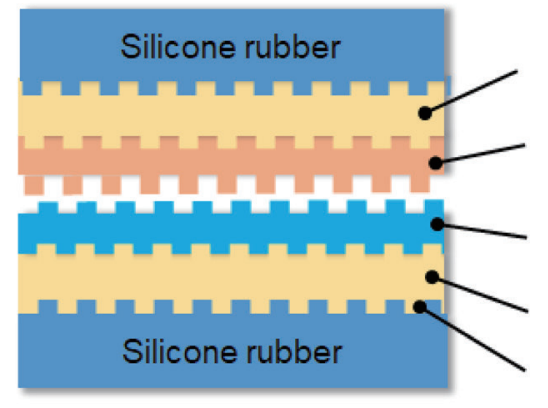

(a)

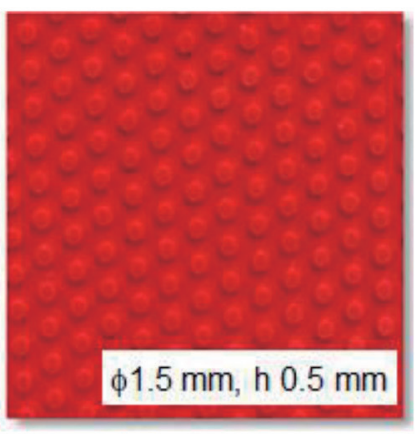

(b)

Fig. 1. (Color online) (a) Structure of TENG used in this study and (b) surface pattern of cylindrical protrusions on silicone rubber. 
PU film with a thickness of $7 \mu \mathrm{m}$ were adhered to the conductive cloth surfaces to make the upper and lower elements. The mechanical and electrical properties of these materials are shown in Table 1. The resistivities of the FEP and PU films are very high, and the hardness of the silicone rubber is small. These elements were used as the TENG with the film surfaces facing each other. Silicone rubber was used as the base material of the triboelectrification films and the electrode to increase the deformation under pressure and the changes in contact area and distance between the mean plane of film surfaces with pressure fluctuations. Furthermore, the silicone rubber surface had cylindrical protrusions that enhanced the changes in contact area and distance between mean planes. The diameter and height of the protrusions were $\phi 1.5$ and $0.5 \mathrm{~mm}$, respectively. The size of the silicone rubber was $50 \times 50 \mathrm{~mm}^{2}$. Electric charge was induced in the conductive cloth by the electrostatic charge on the film surface. When the distance between the mean planes of the film surfaces changed, the charge potential in the conductive cloth changed, and the current could be extracted.

The TENG, as shown in Fig. 1, was packed using an aluminum laminate film with a polyethylene terephthalate/polyamide/aluminum/polyethylene structure to block the water vapor. The TENG package is illustrated in Fig. 2.

\subsection{Environmental evaluation of TENG}

TENGs with and without packaging were evaluated to confirm the effect of environmental temperature and humidity using an environmental chamber, as shown in Fig. 3. The TENG was put on a shaker table that was preloaded with a weight of $42 \mathrm{~N}$. The table was vibrated at

Table 1

Mechanical and electrical characteristics of each material.

\begin{tabular}{lcccc}
\hline Material & Tensile strength $(\mathrm{GPa})$ & Hardness $\left(^{\circ}\right)$ & Dielectric constant & Resistivity $(\Omega \mathrm{cm})$ \\
\hline $\mathrm{FEP}^{(19)}$ & $23.5-37.2$ & - & 2.6 & $>10^{16}$ \\
$\mathrm{PU}$ & - & - & $6.5-7.1$ & $10^{9}-10^{12}$ \\
Conductive cloth & 9.8 & - & - & $<0.05$ \\
Silicone rubber & 9.2 & A 60 & $3-4$ & $>10^{14}$ \\
\hline
\end{tabular}

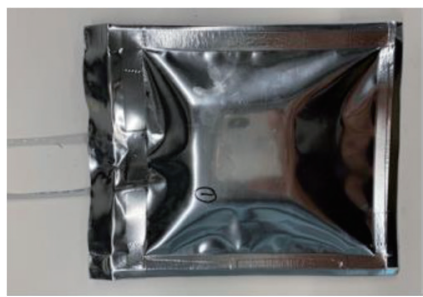

Fig. 2. (Color online) Aluminum laminate packaging of TENG to block humidity.

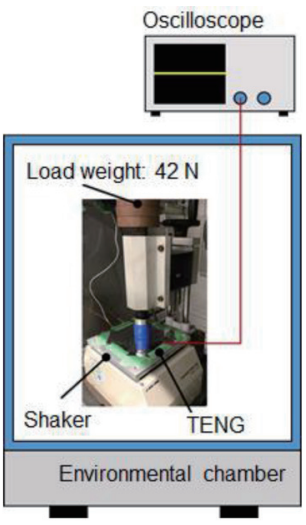

Fig. 3. (Color online) Schematic of experimental setup to evaluate the effect of environmental temperature and humidity on TENG. 
a frequency of $20 \mathrm{~Hz}$ and accelerated at $9.8 \mathrm{~m} / \mathrm{s}^{2}$. The TENG output was monitored using an oscilloscope and the average output voltage was compared at different values of humidity (50-90\% $\mathrm{RH}$ at $\left.25^{\circ} \mathrm{C}\right)$ and temperature $\left(0-80{ }^{\circ} \mathrm{C}\right.$ at $\left.50 \% \mathrm{RH}\right)$.

\subsection{Setup of tire rolling experiment}

The packed TENGs were mounted on the back surface inside the tire using adhesive tape. A racing cart tire (diameter: $210 \mathrm{~mm}$, width: $105 \mathrm{~mm}$ ) was used to evaluate the TENG, as shown in Fig. 4. The TENG output was connected to the wireless data transmitter and the voltage data were received via the receiver. The tire was preloaded with a weight of $150-350 \mathrm{~N}$ and rotated on the moving belt of a treadmill at a speed of $4-16 \mathrm{~km} / \mathrm{h}$ as shown in Fig. 5. The tire pressure was set to $100-250 \mathrm{kPa}$. The generated power was obtained from the voltage across a load resistance of $10 \mathrm{M} \Omega$.

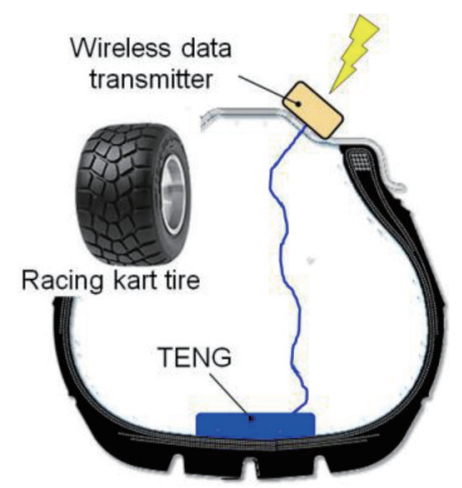

Fig. 4. (Color online) Mounting position of TENG inside the rotating racing kart tire of $210 \mathrm{~mm}$ diameter.

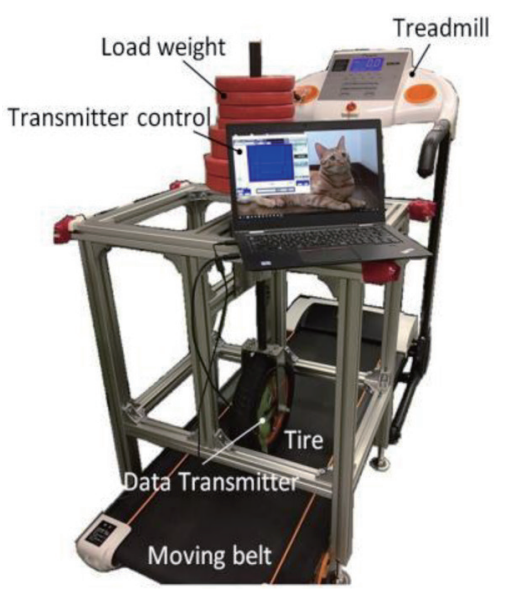

(a)

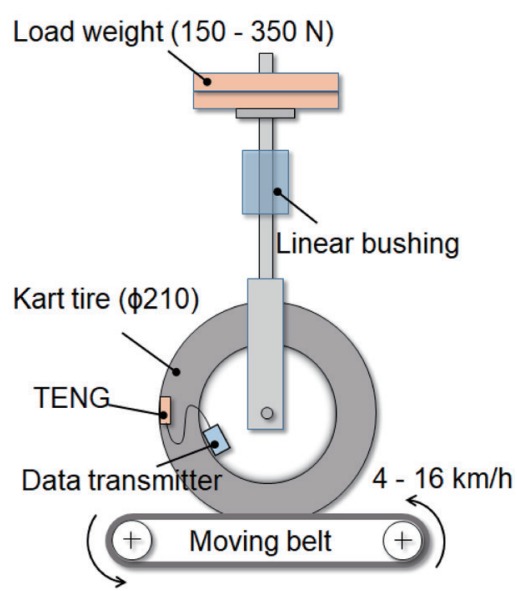

(b)

Fig. 5. (Color online) (a) Evaluation of TENG mounted inside rolling tire on treadmill and (b) schematic of experimental setup. 


\section{Results and Discussion}

\subsection{Dependence of TENG output on environmental temperature and humidity}

The TENG output was measured at different temperatures and humidity environments. A typical TENG output is shown in Fig. 6. The average output voltages, which changed at higher humidity, are shown in Fig. 7. The output voltages of the TENG without an aluminum laminate package decreased as the humidity and temperature increased. However, approximately constant voltage was observed in the packaged TENG despite the changes in external humidity and temperature. Therefore, it was found that the output performance of TENG significantly deteriorates owing to the formation of a water layer on the surface of the triboelectric materials, which depletes the induced charges. ${ }^{(16)}$ Even when the temperature increases, the TENG output decreases owing to the same effect of humidity because the absolute humidity increases. When the TENG is encased in the aluminum laminate package, the output is not affected by the humidity or temperature because the absolute humidity is constant in the package. Considering this result, the proposed TENG element was packed using the aluminum laminate film for mounting in tires.

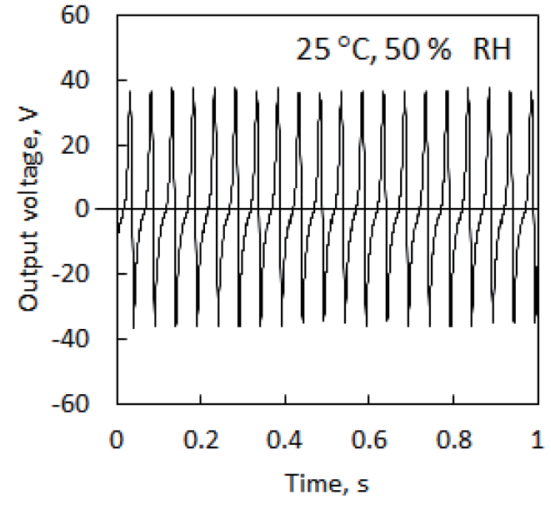

(a)

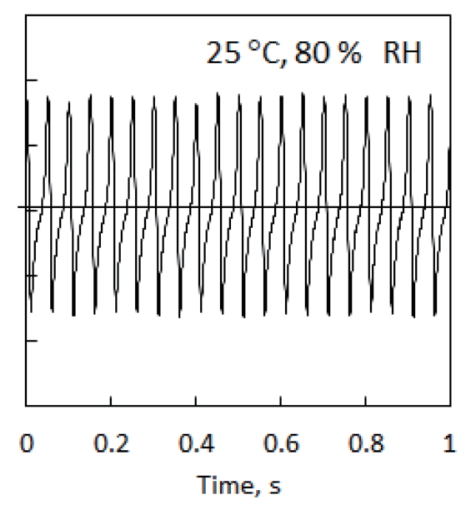

(b)

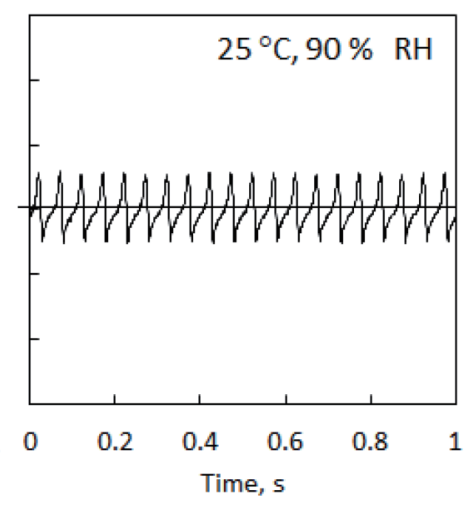

(c)

Fig. 6. Typical output of TENG at different values of environmental humidity, i.e., (a) 50, (b) 80 , and (c) $90 \%$ RH.

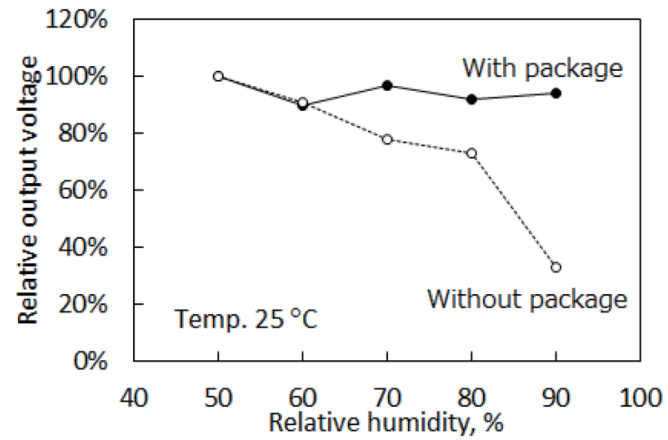

(a)

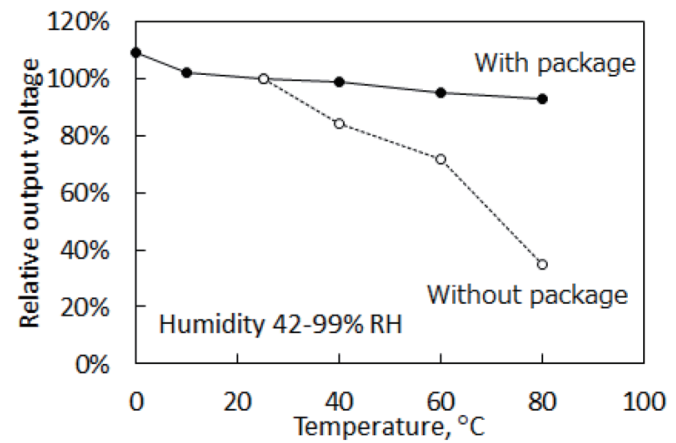

(b)

Fig. 7. (a) Humidity dependence of TENG output and (b) temperature dependence of TENG output. 


\subsection{Output of TENG mounted inside rolling tire}

The performance of the TENG mounted inside the rolling tire was monitored. The typical outputs have been demonstrated in Fig. 8 at different rolling speeds of 8, 12, and $16 \mathrm{~km} / \mathrm{h}$. Two positive peaks and two negative peaks per rotation of the tire were observed. These peaks occurred at the point of contact between the TENG and the tire. To confirm that the output waveform of the TENG is caused by the velocity changes along the radial direction when it comes in contact with a patch of the tire, the radial acceleration component, measured using an acceleration sensor, was compared with the TENG output obtained during the experiment using an automobile tire at a speed of $20 \mathrm{~km} / \mathrm{h}$ on a drum-type rolling resistance tester. The measured TENG output and acceleration sensor output are shown in Fig. 9(a). The waveform of acceleration at the region of contact was found to be similar to that in a previous study. ${ }^{(6)}$ The differential waveform of the TENG output was similar to the acceleration waveform, as shown in Fig. 9(b). This approximation suggests that the output of TENG depends on the velocity component in the radial direction when passing through the contact patch. The dependences of the positive peak voltage of the TENG output on the tire rolling speed, load weight, and tire

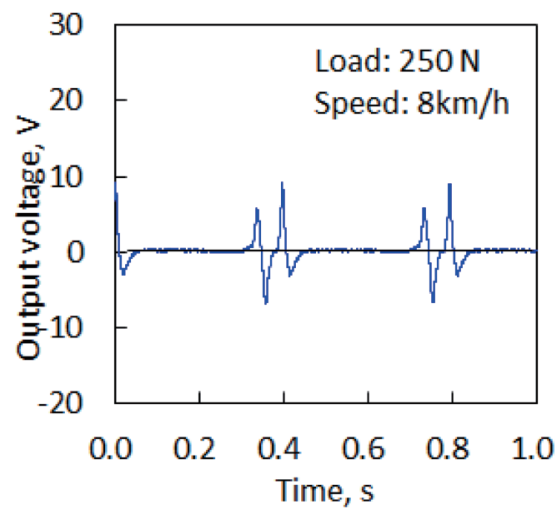

(a)

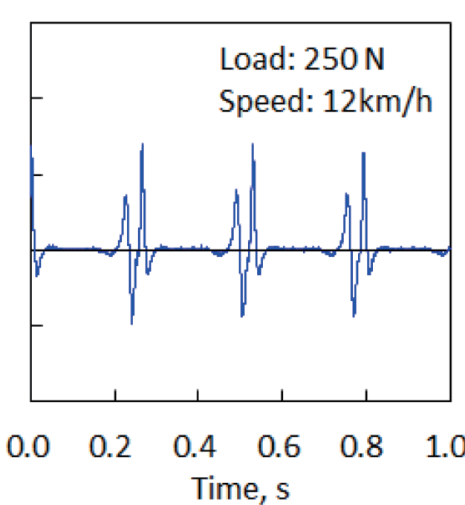

(b)

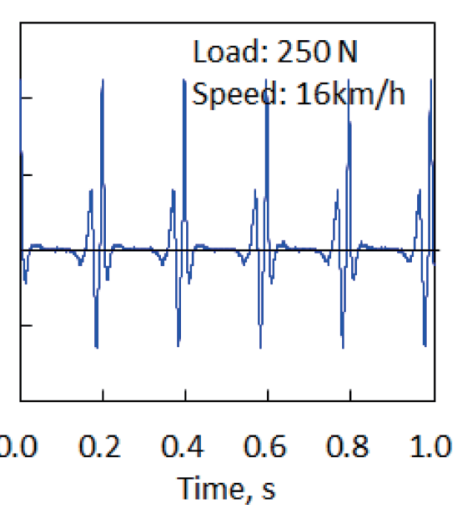

(c)

Fig. 8. (Color online) Typical output of TENG mounted inside rolling tire at different speeds.

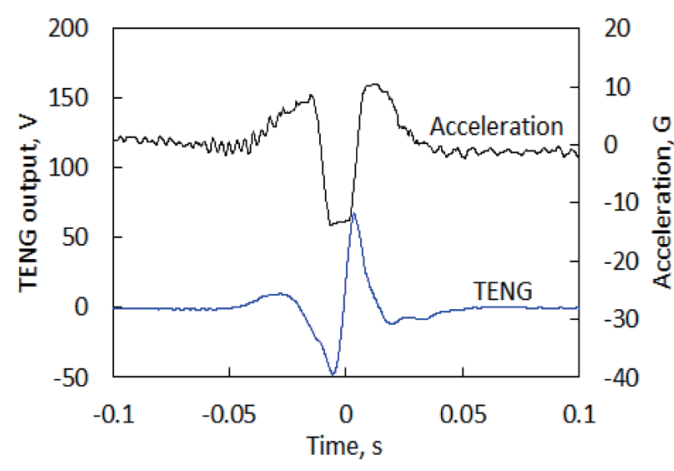

(a)

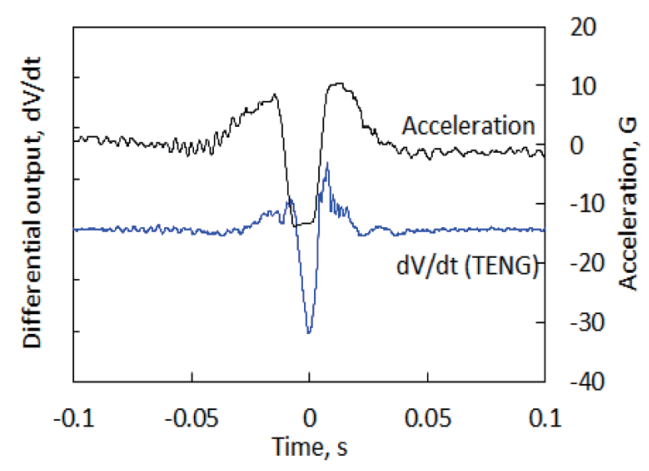

(b)

Fig. 9. (Color online) (a) Acceleration and TENG output. (b) Differential output of TENG and acceleration. 
pressure are illustrated in Figs. 10(a)-10(c), respectively. The TENG output increases with the tire rolling speed because the velocity of tire deformation along the radial direction at the contact patch increases with rolling velocity. It increases with tire load weight because the deformation at the region of contact increases with load weight. In contrast, the TENG output decreases as the tire pressure increases. In this study, we used a racing cart tire with a bias tire structure for the experiment. Therefore, the deformation at the contact patch decreased because the tire bent along the width and expanded. However, it is predicted that automobile tires, which are radial tires, do not expand much even when the tire pressure increases. Because the speed and load of automobile tires are larger than those of cart tires (used in this experiment), it is estimated that the automobile tires exhibit higher voltage. In fact, the positive voltage of the output from the TENG mounted inside the automobile tire at a rolling speed of $20 \mathrm{~km} / \mathrm{h}$ was approximately $70 \mathrm{~V}_{0 \mathrm{p}}$, as shown in Fig. 9.

\subsection{Output power of TENG mounted inside rolling tire}

The charged voltage in the $10 \mu \mathrm{F}$ capacitor was measured using the bridge rectifier. These charged voltages as a function of the rolling speed, rolling duration, load weight, and tire

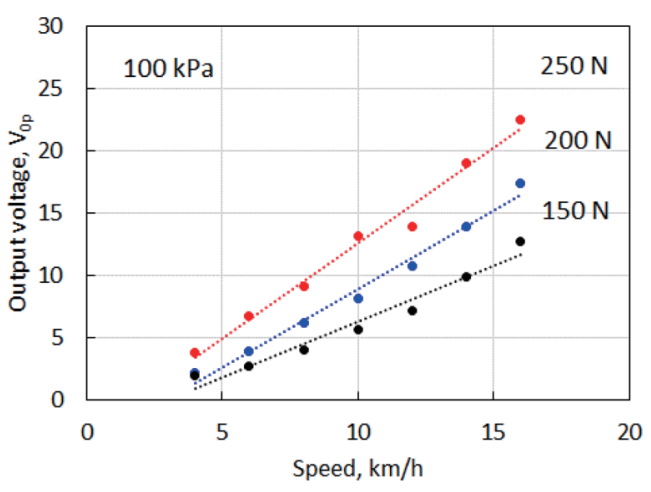

(a)

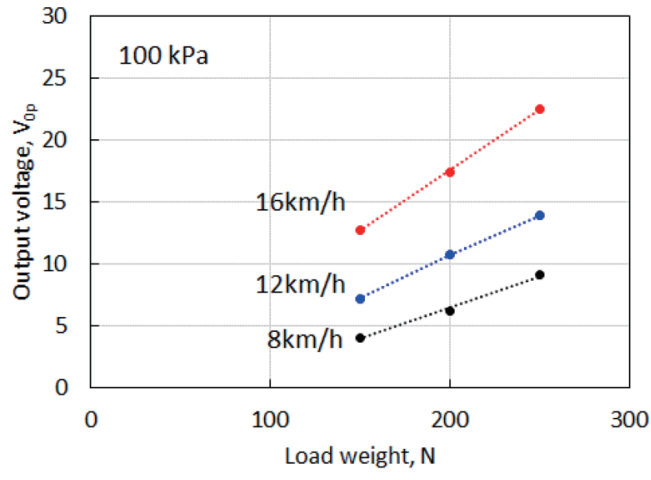

(b)

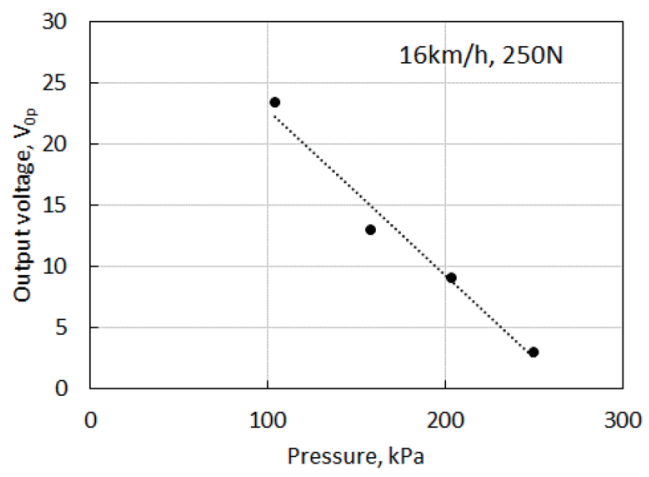

(c)

Fig. 10. (Color online) (a) Rolling speed dependence, (b) load weight dependence, and (c) tire pressure dependence for positive voltage of TENG output. 
pressure of $16 \mathrm{~km} / \mathrm{h}, 10 \mathrm{~min}, 350 \mathrm{~N}$, and $100 \mathrm{kPa}$, respectively, are shown in Figs. 11(a) and 11(b). The charged voltage, found to be approximately $12.5 \mathrm{~V}$, was proportional to the speed. The voltage increased with rolling duration. The charged energy was obtained from the charged voltage, as shown in Fig. 12. The charged energy proportionally increased with rolling duration and became more than $1 \mathrm{~mJ}$ at $15 \mathrm{~min}$. From the slope of the line fitted to the measured data, the power generated by the TENG in the rolling tire under this condition was determined to be approximately $1.0 \mu \mathrm{W}$. If the harvested energy is proportional to the rolling speed, the obtained energy is estimated to be approximately $5.4 \mathrm{~mJ}$ for rolling at the speed of $40 \mathrm{~km} / \mathrm{h}$ for $10 \mathrm{~min}$ by extrapolating the line in Fig. 11(a). In actual car tires, on the basis of the discussion in the previous section, the output power of TENG will be larger than this estimation and it can be concluded that TENG has the potential to be used as a power source for tire sensors.

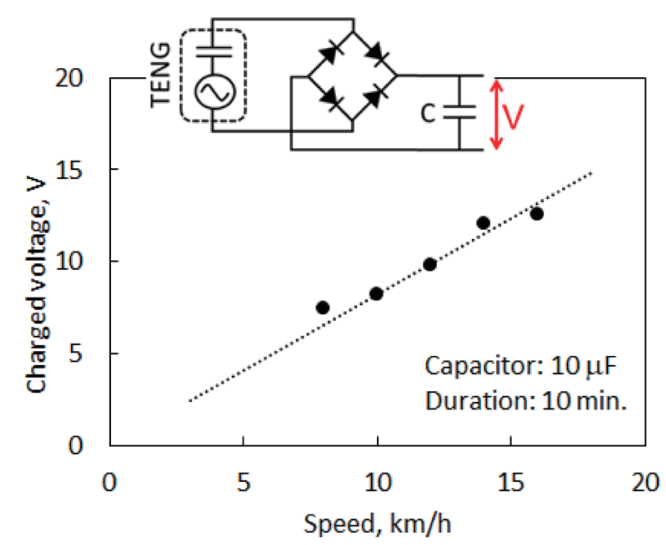

(a)

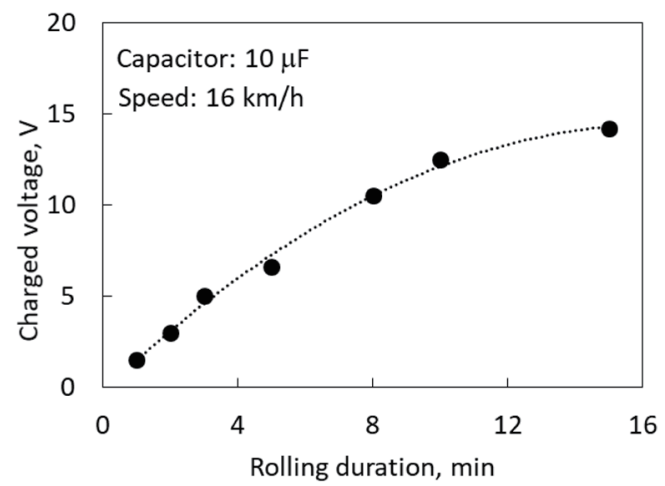

(b)

Fig. 11. (Color online) (a) Charged voltage in capacitor of $10 \mu \mathrm{F}$ as a function of tire rolling speed. (b) Charged voltage in capacitor of $10 \mu \mathrm{F}$ as a function of rolling duration at a rolling speed of $16 \mathrm{~km} / \mathrm{h}$.

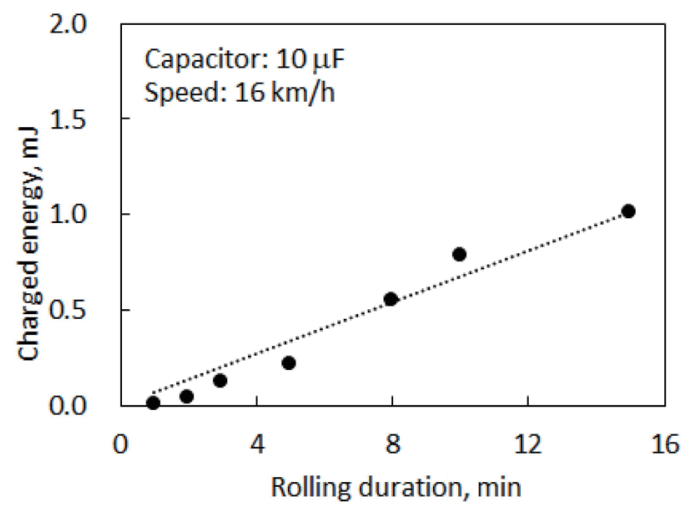

Fig. 12. Energy charged to capacitor as a function of tire rolling duration. 


\section{Conclusions}

In this study, we developed a TENG for intelligent tires. This TENG was attached to the inner surface of a racing cart tire. We evaluated the dependences of the TENG output on the environmental temperature and RH to ensure that the proposed TENG can operate in a wide range of temperature and $\mathrm{RH}$. Then, we prepared TENGs packaged with aluminum laminate film and mounted them on the inner side of the tires. The charged voltage and output voltage of the TENGs were evaluated on a moving belt. The output voltage signals were observed at the region of the contact patch. The signals increased with rotational speed and load weight; however, they decreased with tire pressure. The charged voltage in the $10 \mu \mathrm{F}$ capacitor was found to be approximately $14 \mathrm{~V}$, which corresponds to $1.0 \mathrm{~mJ}$ at the speed of $16 \mathrm{~km} / \mathrm{h}$ after 15 min of tire rotation. These results suggest that the proposed TENG can be used as an energy harvester for the temperature and pressure sensors built into intelligent tires.

\section{Acknowledgments}

This research is supported by the Feasibility Study of Adaptable and Seamless Technology Transfer Program through Target-driven R\&D (A-STEP) from the Japan Science and Technology Agency (JST).

\section{References}

1 H. Askari: Ph.D. thesis of the University of Waterloo (2019). http://hdl.handle.net/10012/14646

2 M. Keck: Proc. IEEE Sensors 2007 (IEEE, 2007) 1299-1302. https://doi.org/10.1109/ICSENS.2007.4388648

3 Q. Zheng, H. Tu, A. Agee, and Y. Xu: Proc. PowerMEMS 2009 (2009) 403.

4 Q. Tang, X. Xia, and X. Li: Proc. Micro Electro Mechanical Systems (MEMS) 2012 (IEEE, 2012) 1273. https:// doi.org/10.1109/MEMSYS.2012.6170389

5 J. Lee and B. Choi: Energy Convers. Manag. 78 (2014) 32. https://doi.org/10.1016/j.enconman.2013.09.054

6 K. B. Singh, V. Bedekar, S. Taheri, and S. Priya: Mechatronics 22 (2012) 970. https://doi.org/10.1016/ j.mechatronics.2012.06.006

7 S. H. Choi, D. P. Chun, D. H. Kwon, S. K. Kim, H. K. Na, and W. J. Lee: US Patent No. 7541916 (2009).

8 Y. -J. Wang, C.-D. Chen, C.-C. Lin, and J.-H. Yu: Micromachines 6 (2015) 312. https://doi.org/10.3390/ mi6030312

9 Y. J. Wang, C. D. Chen, and C. K. Sung: Sens. Actuators, A 159 (2010) 196. https://doi.org/10.1016/ j.sna.2009.12.007

10 Y. Wang, C. Chen, and C. Sung: IEEE/ASME T. Mech. 18 (2013) 754. https://doi.org/10.1109/ TMECH.2012.2183640

11 Y. Mao, D. Geng, E. Liang, and X. Wang: Nano Energy 15 (2015) 227. https://doi.org/10.1016/ j.nanoen.2015.04.026

12 J. Qian, D.-S. Kim, and D.-W. Lee: Nano Energy 49 (2018) 126. https://doi.org/10.1016/j.nanoen.2018.04.022

13 T. Guo, G. Liu, Y. Pang, B. Wu, F. Xi, J. Zhao, T. Bu, X. Fu, X. Li, C. Zhang, and Z. L. Wang: Extreme Mech. Lett. 18 (2018) 1. https://doi.org/10.1016/j.eml.2017.10.002

14 W. Wu, X. Cao, J. Zou, Y. Ma, X. Wu, C. Sun, M. Li, N. Wang, Z. Wang, and L. Zhang: Adv. Funct. Mater. 29 (2018) 1806331. https://doi.org/10.1002/adfm.201806331

15 J. Shen, Z. Li, J. Yu, and B. Ding: Nano Energy 40 (2017) 282. https://doi.org/10.1016/j.nanoen.2017.08.035

16 A. R. Mule, B. Dudem, S. A. Graham, and J. S. Yu: Adv. Funct. Mater. 29 (2019) 1807779. https://doi. org/10.1002/adfm.201807779

17 H. Tani, R. Lu, S. Koaganezawa, and N. Tagawa: Tribologist 63 (2018) 52 (in Japanese). https://doi. org/10.18914/tribologist.17-00015 
H. Tani, J. Ochi, R. Lu, S. Koganezawa, and N. Tagawa: Proc. 2018 JSAE Congress (Autumn) (2018) 047 (in Japanese).

19 Daikin Neoflon technical data sheet, http://www.daikin-korea.co.kr/products/pdf/catalog/GX-27e.pdf (in Japanese).

\section{About the Authors}

Hiroshi Tani received his B.S. and M.S. degrees from Hiroshima University, Japan, in 1984 and 1986, respectively, and his Ph.D. degree from the University of Tokyo, Japan, in 2003. From 1986 to 2008, he worked for Hitachi and Hitach GST. In 2008, he became an associate professor in Kansai University, Japan. Since 2011, he has been a professor at Kansai University. His research interests are in the domain of tribology and triboelectric nanogenerators.

(hrstani@kansai-u.ac.jp)

Mutsuki Sugimoto received his B.E. and M.E. degrees from Yamagata University, Japan, in 2000 and 2002, respectively. Since 2002, he has worked for Sumitomo Rubber Industry Co. His research interests include intelligent tires. (m-sugimoto.az@srigroup.co.jp)

Kazuhisa Fushihara received his B.E. and M.E. degrees from Tohoku University, Japan, in 1991 and 1993, respectively. Since 1993, he has worked for Sumitomo Rubber Industry Co. His research interests include intelligent tires. (k-fushihara.az@srigroup.co.jp)

Yukio Nakano received his B.E. and M.E. degrees from Kobe University, Japan, in 1980 and 1982, respectively. Since 1982, he has worked for Sumitomo Rubber Industry Co. His research interests are in intelligent tires. (y-nakano.az@srigroup.co.jp)

Renguo Lu received his Ph.D. degree from Iwate University, Japan, in 2009. Since 2015, he has been an associate professor at Kansai University. His research interests include tribology.

(r_lu@kansai-u.ac.jp)

Shinji Koganezawa received his Ph.D. degree from Tokyo Institute of Technology, Japan, in 2004. Since 2016, he has been a professor at Kansai University. His research interests are in the field of mechatronics. (skoga@kansai-u.ac.jp)

Norio Tagawa received his B.E. and M.E. degrees from Tohoku University, Japan, in 1975 and 1977, respectively, and his Ph.D. degree from the University of Tokyo, Japan, in 1986. From 1977 to 1997, he worked for NEC. Since 1997, he has been a professor in Kansai University, Japan. His research interests are in the tribology of head-disk interfaces.

(tagawa@kansai-u.ac.jp) 